SRF tatsächlich aktiv bleiben. Sie entwickelt sich zu einem der wesentlichen Akteure des Juba-Friedensprozesses für den Sudan im Jahr 2020.

\title{
Der Weg in den Bürgerkrieg
}

Irgendwann im Zuge des heißen Konfliktes mit dem Sudan und der wegbrechenden Öleinnahmen (eine These, die Alex de Waal, 2014, etabliert hat) entscheidet sich Salva Kiir zur Aufgabe der »big tent«-Politik. Die Auseinandersetzungen innerhalb der SPLM/A werden härter und zunehmend mit offenem Visier ausgetragen. Als auslösender Faktor erweist sich die heikle Frage einer Nachfolgeregelung für Kiir, insbesondere im Hinblick auf die für 2015 angesetzten ersten südsudanesischen Wahlen.

Der vereinbarte Ablauf sieht vor, die Wahlen für Parteivorsitz und Spitzenkandidatur im Rahmen der geplanten dritten National Convention der SPLM im Mai 2013 durchzuführen. Mitte des Jahres 2012 wirft Riek Machar als Erster seinen Hut für eine Gegenkandidatur in den Ring. Ohnehin betrachtet er Kiirs Regentschaft als eine durch den Tod Garangs notwendig gewordene Interimsperiode. Schnell folgen Rebecca Nyandeng und Pagan Amum, die ebenfalls öffentlich ihre Kandidatur deklarieren. Kiir selbst gibt sich hinsichtlich seiner Pläne bedeckt (Johnson, 2014b: 169).

Hinter den Kulissen bereiten sich alle Seiten auf eine sich zuspitzende innerparteiliche Auseinandersetzung vor. Als öffentlichkeitswirksames Instrument dient Kiir dabei eine Anti-Korruptionskampagne. Im Juni 2012 fordert er in einem offenen Brief 75 aktive oder ehemalige Staatsbedienstete auf, alle durch Korruption erschlichenen Gelder, die er auf vier Milliarden US-Dollar schätzt, zurückzuzahlen. Was zu diesem Zeitpunkt wie ein schräger PR-Stunt wirkt, zeigt im Zuge der folgenden politischen Kontroversen schnell seine eigentliche Zielrichtung. Als der Brief seine intendierte politische Druckwirkung nicht zu entfalten droht, folgen konkrete Schritte.

Genau ein Jahr nach dem Verfassen des Briefes entlässt Kiir Finanzminister Kosti Manibe Ngai, einen engen Vertrauten des SPLM-Generalsekretärs Pagan Amum, und den Kabinettsminister Deng Alor unter dem Vorwurf der Korruption.

Diese beiden Entlassungen sind der Startschuss einer sich schnell drehenden Eskalationsspirale. Diese wird noch dadurch befeuert, dass Kiir bereits Ende 2012 dazu übergegangen ist, die zwei wesentlichen Arbeitsgremien der SPLM, das Politbüro und das National Liberation Council, durch Sitzungs- 
verschiebungen und Nichtteilnahmen schleichend stillzulegen. Anfang Juli treffen die Entlassungen dann einen engen Verbündeten von Riek Machar: Taban Deng wird als Gouverneur von Unity State abgesetzt. Er macht sich umgehend an den Aufbau einer oppositionellen Organisation zur politischen Unterstützung für Machar (Johnson, 2014b: 172). Zum endgültigen politischen Auseinanderbrechen der immer schon fragilen Koalition innerhalb der SPLM kommt es schlussendlich am 23. Juli. Kiir entlässt Vizepräsident Machar sowie den Großteil der Regierung und setzt Partei-Generalsekretär Pagan Amum unter Hausarrest. Wenig später wird James Wani, einer der verlässlichsten Kiir-Gefolgsleute, zum neuen Vizepräsidenten ernannt.

Später wird bekannt werden, dass sich ab diesem Zeitpunkt beide Seiten gezielt auf eine bewaffnete Eskalation vorbereiten. Auf Seite Kiirs kommt Paul Malong Awan, zu diesem Zeitpunkt Gouverneur von Northern Bahr el-Ghazal, eine zentrale Rolle zu. Teile der von ihm kontrollierten Dinka-Jugendmiliz Mathiang Anyoor (Boswell, 2019) werden nach Juba transferiert. Peter Nyaba (2019: 176) berichtet, dass diese 3.000 jungen Männer aus Malongs Einflussbereich auf dem privaten Anwesen von Kiir in Luri nahe Juba trainiert werden. Sie beteiligen sich am Aufbau einer weiteren bewaffneten Dinka-Gruppierung, Dotku Beny (Dinka für »wir retten den Chief/Präsident«, Madut Jok: 2017: 24).

Internationale Friedensinitiativen versuchen zu reagieren und die Situation zu beruhigen. Ein Anruf von US Secretary of State John Kerry bei Kiir und Machar bleibt jedoch ohne Erfolg (Vertin, 2019: 260). Die letzte Stufe der Eskalation erfolgt im Dezember. Im Vorfeld eines NLC-Meetings geben eine größere Zahl an SPLM-Granden um Riek Machar am 6. Dezember eine Pressekonferenz, in der sie Kiir der gezielten Anstachelung zu Unruhen beschuldigen und eine unmittelbare Sitzung des Politbüros fordern. ${ }^{9}$ Salva Kiirs Reaktion ist harsch. Zunächst wird Vizepräsident James Wani vorgeschickt, um alle Anschuldigungen abzustreiten und eine Verschiebung des NLC-Meetings zu verlautbaren. Einen Tag später kündigt die parteiinterne Opposition StraBenproteste an, eine Ankündigung, die sie wenig später aufgrund der sich abzeichnenden Eskalation wieder zurückzieht.

Es hilft nichts. Am 15. Dezember brechen in den Baracken im Süden von Juba Schießereien aus. Verwickelt sind unterschiedliche Teile der dort stationieren Tiger Division, der wichtigsten SPLA-Eliteeinheit, die unter ande-

9 https://sudantribune.com/spip.php?article49087 [13-01-2021], der Artikel enthält das an diesem Tag verlesene Presse-Communiqué. 
rem für den Schutz des Präsidenten verantwortlich ist. Die Eskalation macht schnell klar, dass sich die politische Konfrontation zwischen dem Dinka Salva Kiir und dem Nuer Riek Machar entlang ethnopolitischer Linien formieren wird. Jok Madut Jok (2017: loc 2910) berichtet: "At the start of this conflict, perhaps the trigger point of it, one of the Tiger Battalion's officers, a member of the uprising, lined up officers of Dinka origin and executed them himself, sparking the outbreak of violence inside one of the military barracks in Juba«. Am folgenden Tag greifen die Kämpfe auf das SPLA-Hauptquartier in Bilpam über. Kiir verhaftet die Protagonist:innen der Pressekonferenz, darunter Rebecca Nyandeng, Pagan Amum, Deng Alor, Madut Biar Yel und Kosti Manibe, unter dem Vorhalt eines Putsch-Versuches.

Riek Machar ist zu diesem Zeitpunkt bereits im Busch verschwunden, um wenig später die Gründung der SPLM/A-IO (für in opposition) bekannt zu geben. Offenbar wurden auch auf Seite der Opposition schon länger Vorbereitungen für eine bewaffnete Konfrontation getroffen. Es ist allerdings fraglich, wie weit Machar persönlich für diese Vorbereitungen verantwortlich zeichnet. Lam Akols Aussage gegenüber einer nach den Ereignissen eingesetzten Untersuchungskommission der Afrikanischen Union ist vielsagend: »So I don't believe that there was a coup. He [Machar] ran for his life. The mistake I give him is that he took over, a rebellion that was not his." (AUCISS, 2014: 131) Die SPLM/A-IO entwickelt sich eher als eine organisatorische Hülle für all jene militärischen Kräfte, die die Auseinandersetzung innerhalb der SPLA gesucht hatten. Ihr Programm ist rudimentär, ihre inneren Strukturen schwach und autoritär (Young, 2019: 86-87).

Während der folgenden Tage herrscht in Juba nackte Gewalt. DinkaMilizen beginnen im Verein mit SPLA-Truppen eine gezielte Jagd auf Nuer - oder Menschen, die aufgrund ihrer Gesichtstätowierungen für solche gehalten werden. »Diese Tage waren die Hölle«, erzählt ein Freund, der sich damals im Oasis Camp am Nil aufhielt. »Hunderte rannten um ihr Leben. Sie kamen in unseren Compound. Wir ließen sie rein, schlossen die Tür, draußen war Gefechtslärm zu hören. Sie waren in blanker Panik, sprangen in den Fluss, um auf die andere Seite zu schwimmen. Wir hielten sie ab, die meisten wären ertrunken. Einige Stunden später kam ein verlässlicher SPLA-General mit seinen Leuten und brachte sie zum UNMISS-Stützpunkt.« Tatsächlich suchen in diesen Tagen Zigtausende direkten Schutz bei der UN-Peacekeeping Mission, was diese zu einer schnellen Adaption ihrer Protection-of-Civilian (PoC)-Praxis zwingt. 
Die genaue Zahl der Opfer der Gewalt während dieser drei Tage in Juba ist ungeklärt. Internationale Organisationen lassen sich wegen des politischen Charakters derartiger Zahlen auf keine Spekulationen ein. Schätzungen reichen von einigen hundert bis zu 20.000 Toten. John Young (2019: 74) verweist darauf, dass, selbst wenn letztere Zahl zu hoch liegen mag, sie dennoch für die Nuer-Kräfte in der SPLM/A-IO und die mit ihr verbündeten Milizen der White Army ein Faktum darstellen. Sie gelten damit als Messlatte für Vergeltungsaktionen.

Die Gegenoffensive konzentriert sich auf Jonglei und bald auf ganz Greater Upper Nile. Eine Schlüsselrolle in dieser Offensive spielt der Kommandant der in Jonglei stationierten 8. Division der SPLA, Peter Gadet. Er läuft zur SPLM/A-IO über, formiert seine Division unter Nuer-Dominanz neu und schließt sich umgehend mit irregulären Verbänden der White Army zusammen. Am 18. Dezember nimmt Gadet Bor ein und startet einen Marsch auf das etwa 200 Kilometer entfernte Juba. Diese existenziell wirkende Bedrohung bewegt den Hauptverbündeten Kiirs, Ugandas Präsident Yoweri Museveni, zum aktiven Eingreifen. Die bereits an der Grenze stationierten Verbände der ugandischen Armee (UPDF) werden umgehend nach Juba beordert. Die UPDF installiert einen weiten Verteidigungsring im Nordosten, der die überwiegenden Teile Equatorias von dieser ersten Phase des Bürgerkrieges abschirmt. Bor wird im Zuge dieser Operation neben Juba zur zentralen Basis der UPDF.

Die folgenden Kämpfe konzentrieren sich daher auf Unity State und Upper Nile, insbesondere auf die beiden Städte Malakal und Bentiu, der Heimat einer großen Zahl an SPLM/A-IO-Kämpfer:innen. Die Auseinandersetzungen werden mit einer unglaublichen Brutalität geführt, die von vielen im Nachhinein als mit den Kämpfen während des sudanesischen Bürgerkrieges unvergleichbar gewalttätig bezeichnet wird. Die Kontrolle über die beiden Städte wechselt oftmals. Am Ende sind Malakal und Bentiu dem Erdboden gleichgemacht. Zur wohl dramatischsten Eskalation kommt es im April 2014. Am 14. April nehmen Einheiten der SPLA-IO Bentiu und die Schwesterstadt Rubkona ein, vermutlich unter Verstärkung von Milizen der White Army. Am nächsten Tag wird James Koang Chuol, ein Nuer aus Jonglei und erst vor Kurzem zur IO gestoßen, von dieser zum Militärgouverneur ernannt. Er wird aufgrund der nun folgenden Ereignisse, für die er die militärische Letztverantwortung trägt, auf die UN-Sanktionsliste gesetzt.

Am selben Tag, dem 15. April, kommt es zu schweren und systematischen Übergriffen der IO-Truppen gegen Zivilist:innen, die nicht den Nuer zuge- 
rechnet werden. Zunächst werden UN-Posten und das Spital attackiert, danach die Moschee, in die sich mehrere hundert Personen geflüchtet hatten. Dabei handelt es sich vorwiegend um Händler aus Darfur, die offenbar aber dem Justice and Equality Movement (JEM), einer darfurischen Rebellengruppe, die die IO der militärischen Unterstützung der SPLM/A verdächtigt, zugeordnet werden. Eine spätere UNMISS-Untersuchung berichtet, wie ein anfängliches mehrfaches Ausrauben der in der Moschee Schutz suchenden Menschen durch reguläre IO-Kräfte und mit ihnen verbündeter Milizen in einem Massaker kulminiert:

»About half an hour later, a second group of fighters arrived. Among them were individuals in military uniforms, as well as individuals in civilian dress with machetes and firearms. This group went inside the mosque, and demanded money, mobile phones, and other valuable items. Suddenly, several fighters reportedly opened fire.«(UNMISS, 2015: 11)

Allein in der Moschee bleiben 287 Tote zurück.

Der überwiegende Teil der Bevölkerung in Bentiu und Malakal flüchtet in die Lager von UNMISS, die sich an die Errichtung sogenannter PoC-Camps macht. Mitte des Jahres 2015 befinden sich etwa 120.000 Menschen in Bentiu PoC, 48.000 in Malakal PoC. Die Kämpfe um die Städte gehen weiter, wobei zumindest einige der Nuer-Einheiten von Rachegedanken wegen der Massaker in Juba geleitet sein dürften (Young, 2019: 84). Auch wird sichtbar, dass Riek Machar und die IO-Militärführung nur wenig Kontrolle über die Aktivitäten der mit ihnen kämpfenden White Army haben. Zu einer der paradoxen Konstellationen des südsudanesischen Bürgerkrieges gehört, dass der Hauptkontrahent der IO in Greater Upper Nile zu diesem Zeitpunkt die in die SPLA integrierten, von Johnson Olonyi Thabo kommandierten Shilluk-Kräfte sind. Wenige Monate später wird Olonyi selbst zur IO überlaufen.

In Jonglei entwickelt sich die Lage zunehmend unübersichtlich. Der schon einmal reintegrierte Murle-Kommandant David Yau Yau wendet sich erneut von der SPLM/A ab und gründet, mit Unterstützung Khartums, die SSDMCobra Faction. Yau Yaus Miliz strebt an, an die vormals von ihm und George Athor angeführten Murle-Aufstände anzuschließen. Ihm werden aber relativ schnell Friedensverhandlungen angeboten, die im April 2014 in einem Friedensvertrag münden. ${ }^{10}$ Dieses Abkommen entfaltet neben der Wiedereinglie-

10 Agreement on Resolution of the Conflict in Jonglei State between Covernment of the Republic of South Sudan and South Sudan Democratic Movement/Army-Cobra Faction 
derung Yau Yaus in die SPLM/A-Struktur eine weitere nachhaltige Wirkung. Eine bis zum gegenwärtigen Zeitpunkt bestehende Greater Pibor Administrative Area (GPAA) wird eingerichtet, die die Siedlungsgebiete der Murle, Anyuak, Jiye und Kachipo in der Region um Pibor umfasst. Yau Yau wird unmittelbar nach Vertragsabschluss als Chief Administrator der GPAA eingesetzt.

Die nationale politische Ebene kommt ebenfalls in Bewegung. Nach massivem internationalem Druck, der nicht zuletzt von der vagen Hoffnung getragen ist, die Verhafteten könnten eine abschwächende Kraft gegenüber beiden Bürgerkriegsparteien darstellen, entlässt Kiir die ersten sieben der im Dezember 2013 verhafteten Parteigranden aus der Haft. Im Mai darauf folgen Pagan Amum und drei weitere Protagonisten. Sie formieren eine neue, rein politisch agierende Oppositionsbewegung, die SPLM-Former Detainees (SPLMFD), mit dem Ziel, an den für Februar 2015 angesetzten Wahlen teilzunehmen. Allerdings entscheidet sich die Regierung, diese Wahlen aufgrund der anhaltenden Kämpfe - aber auch in Erwartung bevorstehender Friedensverhandlungen - auf unbestimmte Zeit zu verschieben. Dies schmälert allerdings nicht die politischen Ambitionen der SPLM-FD, die sich durch eine strategische Wiederannäherung an die SPLM-IG gezielt im Spiel hält.

Die Ereignisse in Greater Upper Nile und die massive Intervention der UPDF setzen die SPLM/A-IO unter zunehmenden Druck. Ein militärischer Sieg scheint ausgeschlossen. Und mit dem Bentiu-Massaker und dem unkontrollierbaren Auftreten der White Army gerade in Jonglei wurde politisches Kapital nachhaltig verspielt, national wie international. Zur Jahresmitte 2014 beginnen unterschiedliche Verhandlungs- und Gesprächsprozesse zu laufen. Bereits seit Jahresanfang hatten wiederkehrende Waffenstillstandsverhandlungen unter Ägide der IGAD stattgefunden, allerdings mit mäßigem Erfolg. Am 9. Mai 2014 jedoch, unter unmittelbarem Eindruck des Bentiu-Massakers und des nahezu gleichzeitigen Verhandlungserfolges der SPLM/A-IG durch die Einigung mit Yau Yaus Cobra Faction in Jonglei, erklären sich beide Seiten zur Aufnahme von Friedensverhandlungen bereit. ${ }^{11}$ Nach einigem Gezerre und starkem internationalem Druck, ausgeübt vor allem von den USA, wird die SPLM-FD in den Verhandlungsprozess inkludiert. Diese ist vor dem Hintergrund ihrer politischen Schwäche - auf bewaffnete Kräfte konnte sie oh-

(SSDM-SSDA-Cobra) (Yau Yau Agreement), unterzeichnet am 9. Mai 2014, https://ww w.peaceagreements.org/viewmasterdocument/1675.

11 Agreement to Resolve the Crisis in South Sudan, unterzeichnet am 9. Mai 2014 in Addis Abeba, https://www.peaceagreements.org/viewmasterdocument/885. 
nehin nicht zurückgreifen - zum Eingehen einer taktischen Allianz mit der Regierungsfraktion gezwungen, was die Verhandlungsposition der SPLM/AIO zusätzlich unterminiert (Young, 2019: 117-118).

Einige Monate später wird eine erste Prinzipiendeklaration verabschiedet. Sie sieht eine (später verworfene) Machtteilung zwischen einem von der IG gestellten Präsidenten und einem von der IO gestellten Premier in einer für maximal 30 Monate amtierenden Übergangsregierung vor. ${ }^{12}$ Parallel treffen sich die Parteien auf Einladung Tansanias zu Gesprächen zu einer SPLMWiedervereinigung in Arusha, die nach einwöchigen, freundlich wie unverbindlich geführten Debatten ebenfalls in einem Abkommen resultieren. ${ }^{13}$ Dieses legt vage Leitlinien für eine innerparteiliche Demokratisierung fest, etwa eine Amtszeitbeschränkung für den Parteivorsitz. Das Abkommen wird aber schlussendlich ohne jede faktische Bedeutung bleiben.

Im Februar 2015 geraten die Gespräche in eine Krise, wobei sich insbesondere die Einrichtung einer Überwachungskommission, der späteren Joint Monitoring and Evaluation Commission (JMEC), als umstritten erweist. Aus Angst vor der möglichen Installation eines international dominierten Überwachungsgremiums, das die Souveränität und, vor allem, die faktische Macht der südsudanesischen Regierung einschränken könnte, setzt sich die SPLM-IG gegen diese Idee zur Wehr.

IGAD reagiert auf diese Schwierigkeiten, nicht zuletzt auf Anregung der USA, mit einer Verbreiterung der internationalen Abstützung der Mediation unter dem Label IGAD PLUS. Neben der IGAD-Mediation, bestehend aus Äthiopien, Kenia und dem Sudan, umfasst IGAD PLUS die Troika, einige Mitgliedsstaaten der AU sowie die AU Kommission, die UN, die EU, sowie das IGAD Partner Forum. Neben der Begleitung der Verhandlungen legt sich die IGAD PLUS selbst die Überwachung und die Mobilisierung für die Finanzierung des Friedensprozesses auf. Unter dem kumulierten internationalen Druck kommt es zu einer Einigung. Am 17. August 2015 wird das Agreement on the Resolution of Conflict in the Republic of South Sudan (ARCSS) von Kiir, Machar, Amum (für die SPLM-FD) und den Other Political Parties (OPP), einer durch die Crisis in South Sudan, unterzeichnet am 25. August 2014 in Addis Abeba, https://www .peaceagreements.org/viewmasterdocument/1186. 
Verhandlungen faktisch erzwungenen Allianz der zivilen politischen Oppositionsparteien, unterschrieben. Es ist eine Zangengeburt, die dennoch nichts weniger als die nachhaltige Lösung des Konfliktes anstrebt.

Neben der politischen Machtteilung in einem Transitional Government of National Unity (TGoNU), die nicht zuletzt durch die Inklusion der SPLM-FD und ihre Beanspruchung von der Opposition zu füllender Positionen für die Regierungsseite überaus günstig ausfällt, ist ein vages Bekenntnis zum von der SPLM/A-IO geforderten Föderalismus der zentrale Kompromiss des Abkommens. Außer einigen schönen Worten in der Präambel und einem grundsätzlichen Bekenntnis findet sich erstmals die Festlegung, dass ein zukünftiger verfassungsgebender Prozess ein Regierungssystem basierend auf föderalen und demokratischen Prinzipien initiieren sollte. Zudem wird, als eines von gesamt 30 Ministerien, die Einrichtung eines Ministry of Federal Affairs beschlossen. Dennoch sind diese Bekenntnisse für viele nicht weitreichend genug. Speziell die ohnedies wenigen Equatorians in der SPLM/A-IO stehen diesen Zugeständnissen skeptisch gegenüber. Überhaupt findet sich Equatoria im ARCSS-Prozess nicht repräsentiert, weder durch Organisationen noch durch Persönlichkeiten, was in der traditionell eher der SPLM/A-IG zugewandten Region Befremden auslöst.

Die Transitionsphase wird, wie schon in der ersten Prinzipiendeklaration vereinbart, mit 30 Monaten und allgemeinen Wahlen nach zwei Jahren knapp angesetzt. Die Sicherheitssektorreform, und damit der Umgang mit den zwei gegeneinander kämpfenden Armeen, wird vertagt. Ein proportional besetztes Strategic Defence and Security Review Board soll binnen 120 Tagen einen Fahrplan für den bevorstehenden Entwaffnungs- und Reintegrationsprozess (DDR) und eine Sicherheitssektorreform (SSR) erarbeiten. Bewaffnete Verbände außerhalb von SPLA und SPLA-IO sind in dieser Vereinbarung nicht aktiv involviert, sehr wohl aber mitgemeint, was die Exklusivität des Vertragswerkes unterstreicht.

Das ARCSS krankt von Beginn weg an einem für Friedensprozesse letalen Problem: beide Seiten zeigen, aus unterschiedlichen Gründen, keine Ambitionen, es einzuhalten und langfristig umzusetzen. Salva Kiir sieht sich starker interner Opposition ausgesetzt, die, aus militärischer Logik folgerichtig, in einer Situation, wo ein entscheidender Sieg auf dem Schlachtfeld nur mehr als eine Frage der Zeit erscheint, keinen Grund für politische Kompromisse 
gegeben sieht. SPLA-Generalstabschef Paul Malong geriert sich als einer der lautesten Vertreter dieser Denkschule. ${ }^{14}$

Währenddessen nimmt Riek Machar aus einer relativen Position der Schwäche heraus eine Neuformierung der SPLM-IO-Spitze vor: Neben ihm werden Taban Deng und Riek Machars Ehefrau, Angelina Teny, in die engste Führungsebene aufgenommen. Die Konstellation ist pikant. Einerseits sind Angelina Teny und Taban Deng verwandt und phasenweise gemeinsam aufgewachsen. Andererseits ist ihre persönliche Beziehung seit Angelina Tenys Niederlage in der Wahl zum Gouverneur von Unity State im Jahr 2010 und den darauffolgenden Unruhen gelinde gesagt unterkühlt. Auch ist Taban Deng der Hauptkontrahent von Riek Machar um die Führung der südsudanesischen Opposition.

Der eigentliche Todesstoß für das Abkommen erfolgt nicht einmal zwei Monate nach seiner Unterzeichnung. Am 2. Oktober 2015 verordnet Salva Kiir per »Presidential Decree« eine Neuordnung der staatlichen Struktur durch eine Erweiterung auf 28 Gliedstaaten. Angesichts des expliziten Ausschlusses von internen Grenzverschiebungen seitens des Präsidenten in der gültigen Interimsverfassung bedeutet dieser Schritt nicht nur einen wissentlichen Verfassungsbruch. Er untergräbt die vereinbarte Machtteilung auf Ebene der Gliedstaaten, wonach die SPLM/A-IO die Gouverneure von Unity State und Upper Nile State stellen sollte. Zugleich ist der Schritt ein Affront gegenüber dem Föderalismus-Bekenntnis in ARCSS und der politischen Position der IO. Diese favorisiert die Einführung von 21 Staaten entlang einer Aufteilung, die auf die von der britischen Kolonialmacht vor der sudanesischen Unabhängigkeit gezogenen Distriktgrenzen zurückgeht.

Die praktischen Folgen dieser Umstrukturierung sind drastisch. In Upper Nile kommt es durch die neuen Grenzziehungen zu Landenteignungen von Shilluk-Siedlungsgebieten durch Dinka. Es handelt sich vor allem um Gebiete östlich des Nil, die als Eastern Nile State aus dem Territorium des ehemaligen Shilluk-Königreiches herausgebrochen werden. Die Entscheidung führt zum Austritt des Shilluk-Kommandeurs Yoanis Okiech, einem Bruder von Pagan Amum, aus der SPLA. Er gründet die Tiger Faction New Forces (TFNF), mit der er die SPLA, die die Enteignungen durchsetzt, angreift. Diese Kämpfe führen zu einer Massenflucht der Shilluk-Bevölkerung in das PoC-Camp in Ma-

14 Siehe etwa seine vor Vertragsunterzeichnung geäußerte Ablehnung gegen den vereinbarten Machtteilungsschlüssel, https://sudantribune.com/spip.php?article55883 [17-01-2021]. 
lakal, wo sie aber weiterhin Übergriffen von Regierungstruppen ausgesetzt sind (Young, 2019: 145). Letztlich endet die Rebellion mit dem Tod von Okiech im Januar 2017.

Ebenfalls zu Spannungen und vereinzelten Kämpfen kommt es in Western Bahr el-Ghazal. Die Aufteilung des Staates in Wau State und Lol State zerschneidet das Siedlungsland der Fertit, was diese angesichts ihrer relativen numerischen Schwächung in den neuen Teilstaaten de facto von politischen Ernennungen ausschließt. Schon zuvor waren die Fronten in Equatoria in Bewegung geraten, nachdem Kiir im August 2015 den Gouverneur von Western Equatoria, Bangasi Joseph Bakosoro, entlassen hatte. Wenngleich Bakosoro keine relevante militärische Rolle zu spielen in der Lage sein wird, ist seine Entlassung dennoch der Auslöser einer Reihe sich zunehmend segmentierender Konflikte in der Region (ICG, 2016).

Internationaler Druck, vor allem seitens der USA, zwingt Riek Machar dennoch, einzulenken und diese politische Provokation zähneknirschend zu akzeptieren. So entscheidet sich die SPLM/A-IO verspätet, am 27. Oktober 2015, den im September in Addis Abeba ausgehandelten permanenten Waffenstillstand zu unterschreiben. Politisch drängt Taban Deng immer stärker in die Rolle des zentralen Bindeglieds zur Regierung, was bei Riek Machar und seinen Vertrauten erste Befürchtungen über sein mögliches Überlaufen weckt. Der politische Prozess läuft dennoch weiter. Aufgrund von Sicherheitsbedenken schickt die SPLM/A-IO Mitte Dezember zunächst nur ein von Taban Deng geleitetes 150 Mitglieder starkes Vorauskontingent nach Juba. Riek Machar folgt einige Monate später. Ende April trifft er mit SPLA-IOOberkommandierenden Simon Gatwech Dual zum ersten Mal nach Ausbruch des Bürgerkrieges in Juba ein und wird am 26. April formell als Erster Vizepräsident angelobt. Eine Woche später erfolgt die Angelobung der gesamten Übergangsregierung.

Die Formalitäten können jedoch einen konkreten Willen zur Zusammenarbeit nicht ersetzen. Und dieser ist nach wie vor nicht gegeben. Sofort kommt es zu regierungsinternen Streitigkeiten, etwa über die weitere Verfahrensweise mit den Kriegsgefangenen. Zugleich zeigt die SPLA keine Bereitschaft, mit dem Überwachungsmechanismus des Waffenstillstandes, CTSAMM, zusammenzuarbeiten. Stattdessen nehmen die bewaffneten $\mathrm{Zu}-$ sammenstöße zwischen den verfeindeten Truppenteilen an Häufigkeit zu. Am Abend des 7. Juli kommt es schließlich in Juba zu ersten bewaffneten Auseinandersetzungen, als Einheiten der SPLA-IO von Regierungssolda- 
ten attackiert werden. Dies ist allerdings nur ein Vorgeplänkel für den unglückseligen 8. Juli 2016.

Am Morgen jenes Freitags, dem Vortag des fünften Unabhängigkeitsjubiläums, begibt sich Riek Machar zu einem von JMEC vermittelten Treffen mit Salva Kiir und Vizepräsident James Wani Igga in den Präsidentenpalast J1. In der Früh hatten noch beide Seiten zu einer sofortigen Einstellung erster Scharmützel aufgerufen. Ohne Erfolg. Kurz nach dem Eintreffen von Macher bricht vor dem Palast ein heftiges Feuergefecht zwischen den Wachkontingenten von Kiir und Machar aus. Das auf offener Straße ausgetragene Shoot-Out, dessen Spuren auch über vier Jahre später noch an den Wänden gegenüber von J1 sichtbar sind, kostet 150 Menschen das Leben. Riek Machar, zunächst von Salva Kiir höchstpersönlich gegen Kugeln abgeschirmt, gelingt es, zu seiner Basis hinter dem Jebel Kujur zu entkommen. Kurzfristig ist die Lage verworren. Dennoch deuten Augenzeugenberichte, die eine kurzfristige massive Verstärkung des den Präsidenten bewachenden Tiger DivisionKontingents vor dem Gefecht beobachtet haben wollen ${ }^{15}$, auf einen geplanten Charakter dieses Ereignisses hin.

\section{Zusammenbruch und Revitalisierung}

Die darauffolgenden Kämpfe in Juba dauern drei Tage und fordern mehrere hundert Todesopfer. Am 10. Juli kommt es zu einem Angriff auf Machars Hauptquartier, was dessen Entscheidung, in die Demokratische Republik Kongo (DRC) zu fliehen, mitauslösen dürfte. Der Trek in Richtung des Garamba Nationalparks in DRC dauert 30 Tage. Er wird von all jenen unternommen, die die Schlacht in Juba einigermaßen unbeschadet überstehen. Jene SPLA-IO-Kämpfer:innen, die verwundet zurückbleiben, suchen in den beiden von UNMISS bewachten PoC-Sites Schutz. Dies führt zu mitunter langen, persönlichen Trennungen. Im November 2020 treffe ich zufällig einen IO-Soldaten auf seinem ersten Besuch in Juba nach dem damaligen Treck. Plötzlich, gerade als er mir von seinen Erfahrungen auf dem Weg nach DRC berichtet, verliert er jede Contenance. Er springt auf und umarmt einen neu Hinzukommenden, minutenlang, unter Tränen. Es ist sein Bruder, der nach Verwundungen in Juba im PoC Camp in Juba zurückbleiben musste. Es war ihr erstes Wiedersehen nach über vier Jahren. 